\title{
Refractive outcomes and optical quality after implantation of posterior chamber phakic implantable collamer lens with a central hole (ICL V4c)
}

\author{
Huamao Miao ${ }^{\dagger}$, Xun Chen ${ }^{\dagger}$, Mi Tian, Yingjun Chen, Xiaoying Wang ${ }^{*}$ and Xingtao Zhou ${ }^{*}$
}

\begin{abstract}
Background: To investigate refractive outcomes and optical quality after implantation of posterior chamber phakic implantable collamer lens with a central hole (ICL V4c) to correct high myopia.

Methods: Sixty seven eyes of 38 patients who underwent ICL V4C implantation were enrolled. The mean preoperative spherical equivalent (SE) was $-12.44 \pm 3.15 \mathrm{D}$ (range: -6.63 to $-20.50 \mathrm{D}$ ). The refractive outcomes and optical quality of the eyes at postoperative 1 and 3 months were evaluated and compared.

Results: At 3 months postoperatively, the mean safety and efficacy indexes were $1.33 \pm 0.22$ and $1.14 \pm 0.23$, respectively. The mean SE was $-0.32 \pm 0.52 \mathrm{D}$; no patient lost 1 or more lines of corrected distance visual acuity (CDVA), 13\% remained unchanged, 45\% gained 1 line and $42 \%$ gained 2 or more lines. The mean modulation transfer function cutoff frequency ( $\left(\mathrm{MTF}_{\text {cutoff }}\right.$ ), Strehl in two dimensions ratio, and objective scatter index (OSI) were $38.20 \pm 9.96$ cycles per degree, $0.21 \pm 0.06$, and $1.00 \pm 0.73$, respectively. No significant difference was found in any of the above parameters $(P>0.05)$ between 1 and 3 months. The postoperative intraocular pressure (IOP) did not change when compared with preoperative values $(P>0.05)$.
\end{abstract}

Conclusions: ICL V4C implantation is a safe, effective, and stable solution for high myopia. Patients will acquire high and stable postoperative optical quality. ICL V4C implantation has little influence on IOP.

Keywords: Myopia, Phakic intraocular Lens, V4c, Refractive outcomes, Optical quality, Intraocular scattering, Intraocular pressure

\section{Background}

The prevalence of myopia in China is very high. Most patients seeking refractive surgery treatments suffer from moderate to high myopia. Laser corneal refractive surgeries to correct myopia have gained wide acceptance in China. However, the surgery is not safe for patients with high level of refractive error and relatively thin corneas, as these conditions could increase the risk of postoperative corneal ectasia [1]. Phakic intraocular lens implantation could correct a wider range of refractive

\footnotetext{
*Correspondence: doctxiaoyingwang@163.com; doctzhouxingtao@163.com †Huamao Miao and Xun Chen contributed equally to this work. Department of Ophthalmology, Eye and ENT Hospital of Fudan Universtity, NHC Key Laboratory of Myopia (Fudan Universtity), No.83 FenYang Road, Shanghai 200031, People's Republic of China
}

errors and avoid decreases in corneal thickness; therefore, it would be a good option for patients with these conditions. Even in eyes with keratoconus, the posterior chamber toric phakic ICL was found to be effective in correcting high myopic astigmatism [2].

The posterior chamber phakic implantable collamer lens with a central hole (ICL V4c) is a newly developed kind of ICL, which is designed with a $360 \mu \mathrm{m}$ central hole in the central optical zone. The advantage of the ICL V4c implantation is that it is less traumatic than the conventional ICL (without a central hole) implantation. Experimental and clinical studies conducted in non-Chinese countries have proved that, by improving the circulation of aqueous humour to the anterior surface of the crystalline lens, the specially designed central hole helps to maintain the

(c) The Author(s). 2018 Open Access This article is distributed under the terms of the Creative Commons Attribution 4.0 International License (http://creativecommons.org/licenses/by/4.0/), which permits unrestricted use, distribution, and 
intraocular pressure (IOP) without additional peripheral iridotomy $[3,4]$. However, the peripheral iridotomy is an inevitable procedure in conducting conventional ICL (without a central hole) implantation. In addition, secondary anterior segment cataract formation is the main concern after implantation of a conventional ICL $[5,6]$. According to the previous experimental results, the ICL $\mathrm{V} 4 \mathrm{c}$ also holds the potential to reduce the risk of cataract formation [7-9]. Thus, it is considered that the ICL V4c could be a promising alternative to the conventional ICL for myopia correction.

This prospective study reported the refractive outcomes and optical quality in the 3 months after ICL V4c implantation in a group of Chinese adults with high myopia.

\section{Methods}

\section{Subjects}

In this prospective non-randomized study, 67 eyes of 38 consecutive patients (9 unilateral and 29 bilateral patients), 10 of which were male and 28 of which were female, were examined. The mean age was $28.61 \pm$ 6.10 years (range: 18 to 40 years). Preoperatively, all the patients underwent routine ophthalmic examinations at the Refractive Surgery Center of the Department of Ophthalmology, Eye and ENT Hospital of Fudan University (Shanghai, People's Republic of China) and met the surgical requirements for ICL V4c (STAAR Surgical Company, Monrovia, California, USA) implantation. Inclusion criteria were: aged between 18 and 40 years, spherical refraction error between $-6.00 \mathrm{D}$ and -20.00 $\mathrm{D}$, astigmatism of up to $-5.00 \mathrm{D}$, corrected distance visual acuity (CDVA) of 20/40 or better, anterior chamber depth of $\geq 2.8 \mathrm{~mm}$, and endothelial cell density of $\geq 2000$ cell $/ \mathrm{mm}^{2}$. Exclusion criteria were: a history of certain ocular diseases (suspicion of keratectasia, cornea or lens opacity, retinal detachment, glaucoma, macular degeneration, or neuro-ophthalmic disease), a history of ocular surgery, inflammation or trauma, and systemic disease.

This study adhered to the Declaration of Helsinki and was approved by the Ethical Committee Review Board of Fudan University Eye and ENT Hospital. All patients gave written informed consent after the possible risks and benefits of the study were explained.

\section{ICL V4c}

The ICL V4c is a plate-haptic single-piece intraocular lens made of collamer. It has a central convex-concave optical zone and incorporates a forward vault to minimize contact with the crystalline lens. A $360 \mu \mathrm{m}$ central hole was included to improve aqueous humor circulation, which eliminates the need for preoperative laser peripheral iridotomy [7]. The ICL V4c corrects $0.50 \mathrm{D}$ to $-18.00 \mathrm{D}$ myopic spherical refraction and up to $-5.00 \mathrm{D}$ cylindrical refraction. There are 4 sizes: $12.1 \mathrm{~mm}, 12.6 \mathrm{~mm}, 13.2 \mathrm{~mm}$, and $13.7 \mathrm{~mm}$. Power calculation of the ICL V4c was performed by the manufacturer using a modified vertex formula, according to the provided preoperative refractive parameters. The size of the implanted ICL V4c was determined based on the white-to-white horizontal corneal diameter and anterior chamber depth. Toric ICL V4c is designed to correct both spherical and cylindrical diopters. In the present study, toric ICL V4c was implanted in 30 eyes, with a mean preoperative cylindrical diopter of $-2.64 \pm 1.06 \mathrm{D}$ (range, $-1.25 \mathrm{D}$ to $-5.00 \mathrm{D}$ ); the other 37 eyes chose the ordinary ICL V4c and the mean preoperative cylindrical diopter was $-0.91 \pm 0.54 \mathrm{D}$ (range, $-0.25 \mathrm{D}$ to $-2.50 \mathrm{D}$ ).

\section{Surgical technique}

ICL V4c implantation procedures were performed by two experienced surgeons (XZ and XW). Pupils were dilated before surgery. After injection of $1 \%$ sodium hyaluronate into the anterior chamber via a puncture site at the 6 o'clock position of the cornea, an ICL V4c was implanted via a $3.0 \mathrm{~mm}$ temporal corneal incision using an injector cartridge and then was placed in the posterior chamber. After that, the viscoelastic surgical agent was completely removed using a balanced salt solution, and a miotic agent was instilled. Postoperative medications included antibiotics eye drops, non-steroidal anti-inflammatory eye drops, steroidal eye drops, and artificial eye drops. At 1 and 3 months postoperatively, the following parameters were collected: manifest refraction, uncorrected distance visual acuity (UDVA), CDVA, IOP measured with a non-contact tonometer (NCT; Canon, Japan), retinal image quality, and intraocular scattering. Four patients did not attend the 1-month follow-up. The safety index was calculated as the ratio of the CDVA at 3 months to the corresponding preoperative CDVA, and the efficacy index was the ratio of the UDVA at 3 months to the preoperative CDVA [10].

\section{Retinal image quality and intraocular scattering measurement}

Retinal image quality and intraocular scattering were objectively measured using a double-pass optical quality analysis system (OQAS II; Visiometrics, Terrassa, Spain). Before the measurements, the cylindrical diopter of $0.50 \mathrm{D}$ or higher should be corrected using an external lens and the spherical diopter was automatically corrected by the double-pass system. The system has been used in our clinical practice to evaluate optical quality in myopic patients and after corneal refractive surgeries, and the same methods were applied in the present study [10-12]. In brief, a two-dimension modulation transfer function (MTF) profile was calculated from the image of a light source $(780 \mathrm{~nm}$ laser diode) reflected on the 
retina using the Fourier transform. Five representative indexes were derived from the MTF profile for retinal image quality evaluation, including MTF cutoff frequency $\left(\mathrm{MTF}_{\text {cutoff }}\right)$, Strehl in 2 dimensions (Strehl2D) ratio, and OQAS values (OV) at 3 levels of contrast.

$\mathrm{MTF}_{\text {cutoff }}$ represents the spatial frequency at which the MTF value is 0.01 . Theoretically, an $\mathrm{MTF}_{\text {cutoff }}$ of $30 \mathrm{cy}-$ cles per degree (cpd) usually corresponds to $20 / 20$ visual acuity, and the maximum value is no more than $60 \mathrm{cpd}$ [13]. The Strehl2D ratio is a more comprehensive index for evaluating optical quality. It is the ratio between the aberrated eye and the ideal aberration-free eye in the MTF profiles, and the value ranges between 0 and 1.0. OVs of 100,20 , and $9 \%$ were calculated as the spatial frequencies at the MTF values of $0.01,0.05$, and 0.1, respectively, divided by $30 \mathrm{cpd}$ [13]. These are normalized values that are comparable to the standard decimal measurement for visual acuity. Higher values indicate higher optical quality. The above parameters are independent of retinal and neural factors.

The double-pass system uses objective scatter index (OSI) as an objective parameter to estimate intraocular scattering. The OSI is calculated as the ratio of the amount of light in the peripheral zone (an annular area of 12 and $20 \mathrm{~min}$ ) to the central zone (central peak of $1 \mathrm{~min}$ of arc) of the retinal image [13-15]. An OSI value of less than 1.0 indicates low scattering.

\section{Statistical analysis}

Refractive outcome graphs were plotted using Microsoft Excel according to the refractive outcomes at 3 months in all the 67 eyes of the 38 patients. All statistical analyses were performed using the software Statistical Package for the Social Sciences (SPSS) Version 20.0 (SPSS, Chicago, IL, USA). The Kolmogoro-Smirnov test was used to determine if a variable is normally distributed. If both eyes were recruited in the study, only one eye was selected at random for comparison analysis, at last, 35 eyes of the 35 patients whose data were available in both follow-ups were selected. According to each variable's distribution, a paired $t$ test or Wilcoxon signed-rank test was chosed to compare the parameters between time points ( 1 and 3 months postoperatively).

\section{Results}

Preoperatively, the mean spherical equivalent (SE) of the 67 eyes was $-12.44 \pm 3.15 \mathrm{D}$ (range: -6.63 to -20.50 $\mathrm{D}$ ), the mean sphere was $-11.60 \pm 3.05 \mathrm{D}$ (range: -6.25 to $-19.25 \mathrm{D})$, the mean cylinder was $-1.69 \pm 1.18 \mathrm{D}$ (range: -0.25 to $-5.00 \mathrm{D}$ ), and the mean $\log \mathrm{MAR}$ CDVA was $0.04 \pm 0.08$ (range: -0.08 to 0.30 ).

At 3 months postoperatively, the mean values of the safety and efficacy indexes of all 67 eyes were $1.33 \pm 0.22$ and $1.14 \pm 0.23$, respectively, and the mean logMAR
CDVA and UDVA were $-0.08 \pm 0.07$ and $-0.01 \pm 0.09$, respectively. The refractive outcomes of all 67 eyes at 3 months postoperatively were summarized in Fig. 1 . All the eyes had a CDVA or UDVA of $20 / 40$ or better at 1 and 3 months (Fig. 1a). Eyes with a CDVA of 20/20 or better increased from preoperative 70 to $94 \%$ at 3 months postoperatively. None of the 67 eyes lost 1 or more lines of CDVA, $13 \%$ remained unchanged, $45 \%$ gained 1 line, and $42 \%$ gained 2 or more lines (Fig. 1b). A scatterplot and the best linear fit line $\left(r^{2}=0.9979\right)$ of the attempted versus the achieved SE correction are shown in Fig. 1c. Of the 67 eyes, $92.54 \%$ were within $\pm 1.00 \mathrm{D}$ (Fig. 1c, green lines) and $100 \%$ were within $\pm 2.00 \mathrm{D}$ of the desired SE refraction (Fig. 1c, purple lines). At 3 months, 72,95 , and $100 \%$ of the eyes had an SE refraction within $\pm 0.5, \pm 1.0$, and \pm 2.0 D, respectively (Fig. $1 \mathrm{~d}$ ), and 60 , 83 , and $99 \%$ had astigmatism within $\pm 0.5, \pm 1.0$, and \pm 2.0 D, respectively (Fig. 1e). The mean SE increased from preoperative $-12.44 \pm 3.15 \mathrm{D}$ to $-0.30 \pm 0.52 \mathrm{D}$ at 3 months, and the SE refraction barely changed from 1 to 3 months (Fig. 1f). When the refractive parameters were compared between 1 and 3 months, no significant differences were observed regarding refractive sphere, refractive cylinder, SE, logMAR UDVA or CDVA, safety index, or efficacy index $(P>0.05$; Table 1$)$.

At 3 months postoperatively, the mean $\mathrm{MTF}_{\text {cutoff }}$ was $38.20 \pm 9.96 \mathrm{cpd}$ (range: 13.14 to $55.59 \mathrm{cpd}$ ), the mean Strehl2D ratio was $0.21 \pm 0.06$ (range: 0.08 to 0.37 ), and the mean OSI was $1.00 \pm 0.73$ (range: 0.24 to 3.47 ) in all 67 eyes. The mean OVs at 100, 20, and 9\% contrasts, were $1.27 \pm 0.33$ (range: 0.44 to 1.85 ), $1.27 \pm 0.40$ (range: 0.39 to 2.13 ), and $1.24 \pm 0.43$ (range: 0.37 to 2.34 ), respectively. No significant difference was found between 1 and 3 months postoperatively in any of the above optical quality-related parameters $(P>0.05$; Table 2$)$.

The mean intraocular pressure of all the eyes was $15.30 \pm 2.93 \mathrm{mmHg}$ (range: 8.0 to $21.8 \mathrm{mmHg}$ ) preoperatively and $14.19 \pm 2.62 \mathrm{mmHg}$ (range: 9.3 to $20.5 \mathrm{mmHg}$ ) at 3 months postoperatively. No significant difference was observed between time points $(P>0.05$; Fig. 2$)$.

\section{Discussion}

The newly developed ICL V4c has been reported in recent studies to produce encouraging clinical and experimental outcomes [3, 4, 16-27]. However, little is known about ICL V4c implantation in Chinese patients. Our study presented the visual outcomes, retinal image quality, intraocular scattering, and IOP results in 3 months after ICL V4c implantation in patients with high myopia.

In our study, none of the subjects lost their preoperative CDVA. Additionally, $87 \%$ of eyes gained 1 or more lines of CDVA, and the mean safety and efficacy indexes were over 1.1. In previous studies regarding visual outcomes 3 to 12 months after ICL V4c implantation, the mean values of 


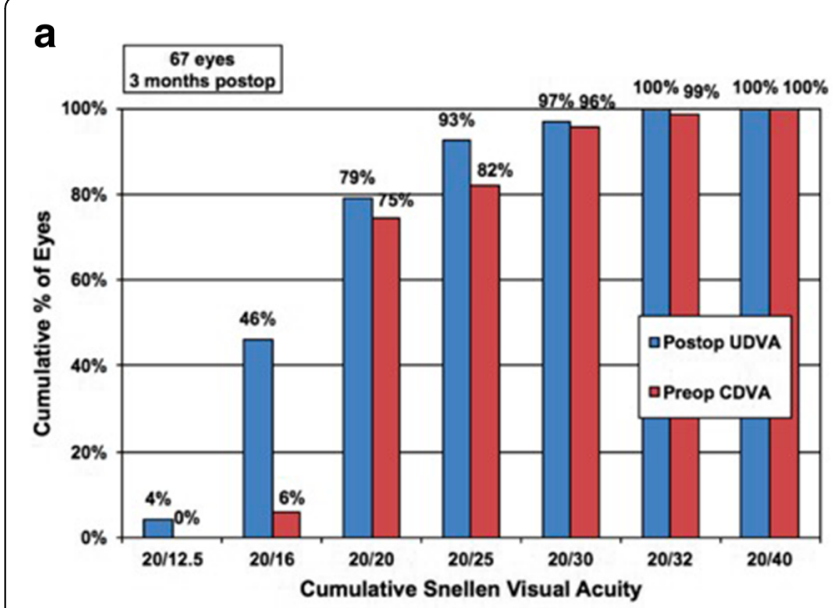

C
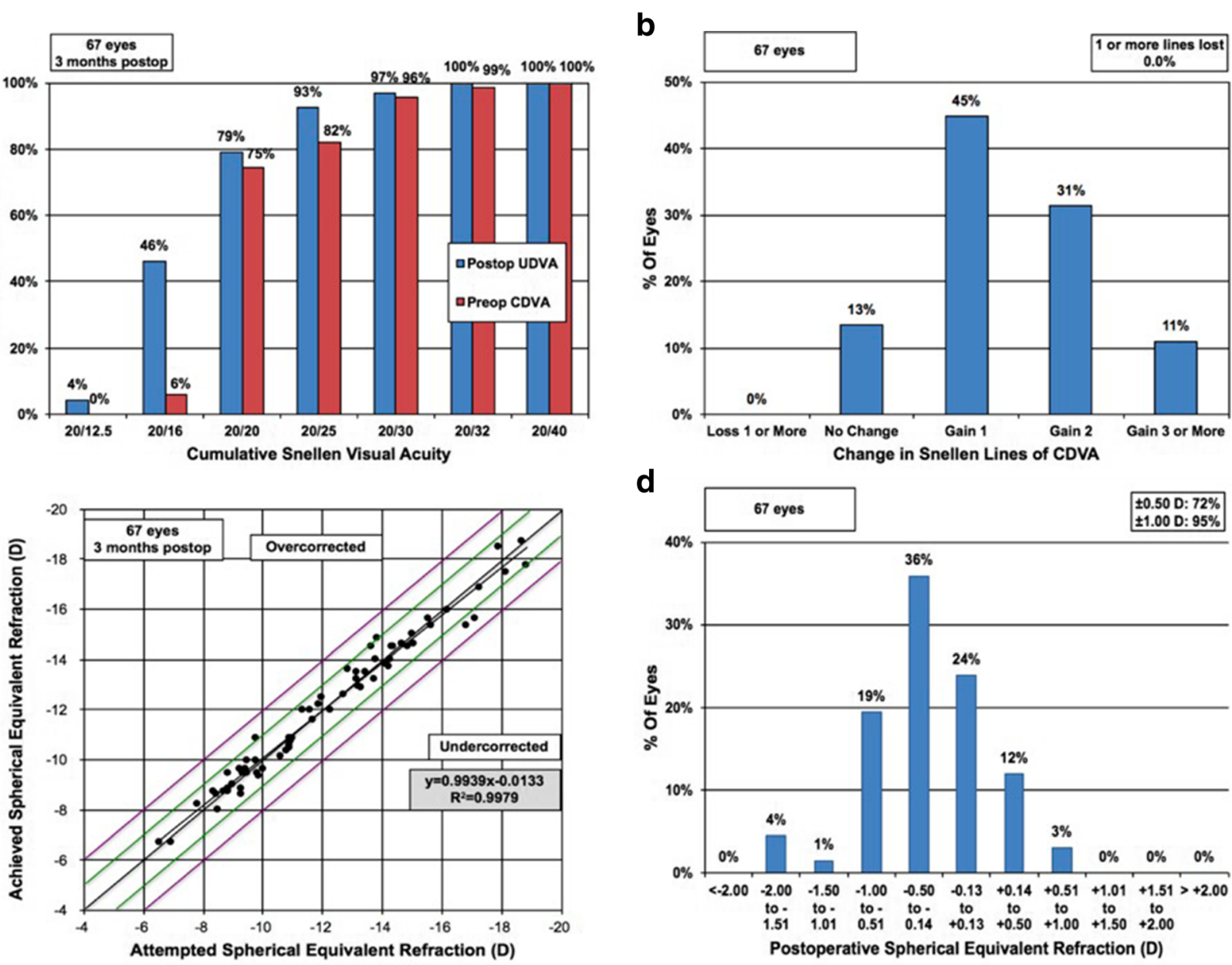

d
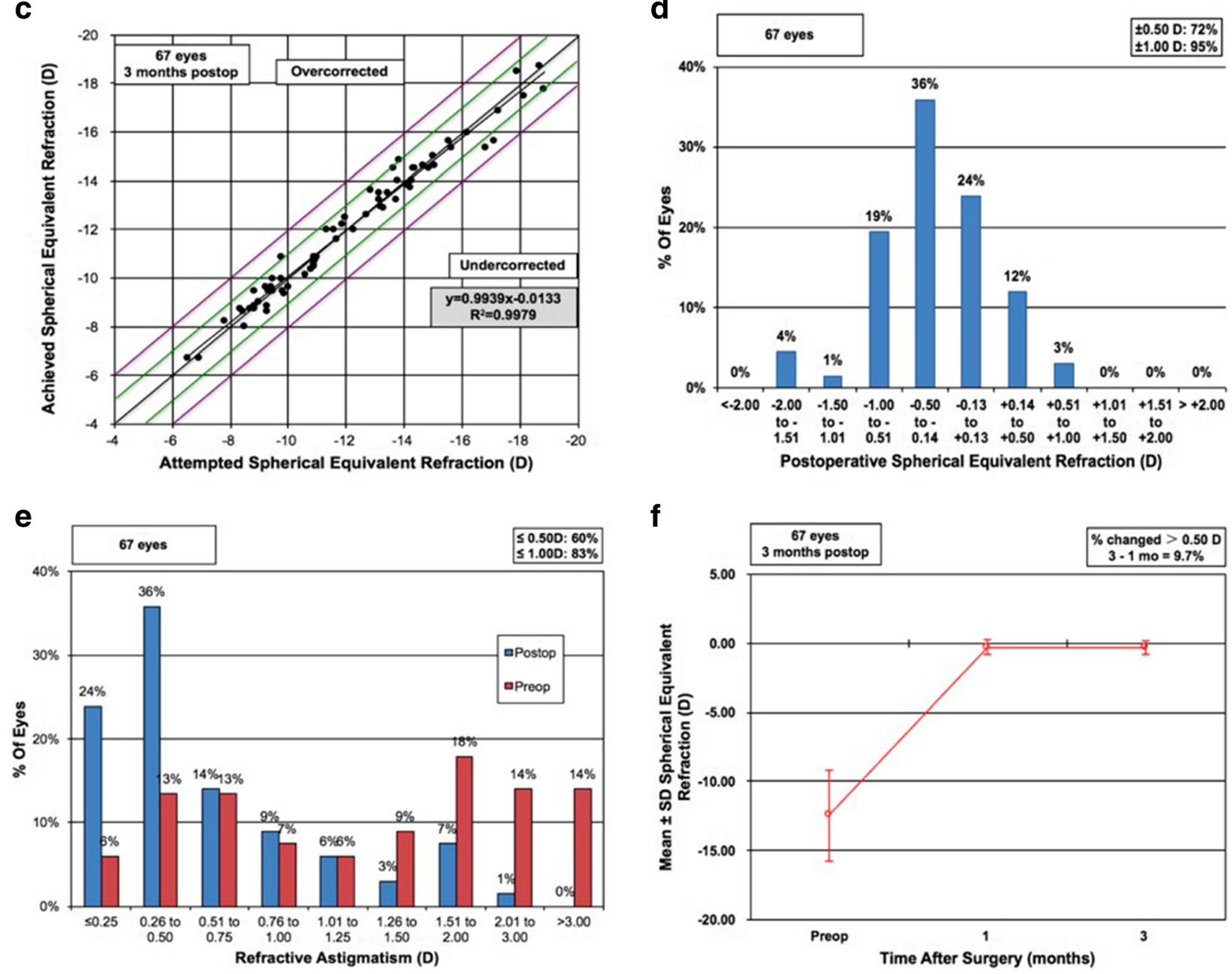

Fig. 1 Refractive outcomes at 3 months postoperatively in 67 eyes with high myopia after implantation of posterior chamber phakic implantable collamer lens (ICL V4c) with a central hole, including (a) postoperative uncorrected distance visual acuity, (b) change in corrected distance visual acuity, (c) attempted versus achieved spherical equivalent, (d) spherical equivalent refractive accuracy, (e) refractive astigmatism, and (f) stability of spherical equivalent refraction. UDVA = uncorrected distance visual acuity; CDVA = corrected distance visual acuity; $D=$ diopters; Postop = postoperative; Preop $=$ preoperative; $\mathrm{SD}=$ standard deviation; $\mathrm{mo}=$ months 
Table 1 Refractive Outcomes at 1 and 3 Months after ICL V4C Implantation for Correcting High Myopia. $(n=35)$

\begin{tabular}{|c|c|c|c|c|c|}
\hline \multirow[t]{2}{*}{ Parameters } & \multicolumn{2}{|c|}{1 Month } & \multicolumn{2}{|c|}{3 Months } & \multirow[t]{2}{*}{ P } \\
\hline & Mean & SD & Mean & SD & \\
\hline Sphere (D) & 0.09 & 0.56 & 0.10 & 0.54 & 0.906 \\
\hline Cylinder (D) & -0.73 & 0.54 & -0.71 & 0.52 & 0.786 \\
\hline SE (D) & -0.27 & 0.51 & -0.26 & 0.47 & 0.793 \\
\hline $\log M A R$ UDVA & -0.01 & 0.11 & -0.01 & 0.09 & 0.580 \\
\hline $\log M A R$ CDVA & -0.06 & 0.09 & -0.08 & 0.08 & 0.071 \\
\hline Efficacy index & 1.11 & 0.22 & 1.13 & 0.24 & 0.797 \\
\hline Safety index & 1.25 & 0.20 & 1.31 & 0.22 & 0.060 \\
\hline
\end{tabular}

ICL V4C Posterior Chamber Phakic Implantable collamer lens With a Central Hole, $n$ number of eyes, $S D$ standard deviation, $S E$ spherical equivalent refraction, $D$ diopters, logMAR the logarithm of the minimal angle of resolution, UDVA uncorrected distance visual acuity, CDVA corrected distance visual acuity

the two indexes were over 1.0, and approximately 20 to $40 \%$ eyes gained 1 or more lines of CDVA [16-20]. The significant improvement in postoperative visual acuity in our study supports the safety and efficacy of ICL V4c implantation for myopia correction. Patients enrolled in the previous studies had spherical refraction ranging from -2.75 to $-17.50 \mathrm{D}$, astigmatism of $-3.00 \mathrm{D}$ or less, and a mean SE ranging from -7.36 to $-9.32 \mathrm{D}$ [16-20], while the values were relatively higher in our study, with spherical refraction ranging from -6.25 to $-19.25 \mathrm{D}$, a maximum astigmatism of $-5.00 \mathrm{D}$, and a mean SE of - 12.44 D. In addition, part of the patients with astigmatism in the current study did not choose the toric ICL V4c. Even so, the results showed relatively satisfying predictability, with $95 \%$ or $83 \%$ of the eyes had SE or astigmatism within $\pm 1.00 \mathrm{D}$ at 3 months. Additionally, the refractive parameters, including visual acuity and manifest refraction, showed identical results at 1 and 3 months postoperatively, suggesting that patients' refractive status could be stabilized soon after ICL V4c implantation.

Particular attention was paid to optical quality after refractive surgeries. Both the conventional ICL and the ICL $\mathrm{V} 4 \mathrm{c}$ implantation procedures provide good optical and

Table 2 Optical Quality at 1 and 3 Months after ICL V4C Implantation for Correcting High Myopia $(n=35)$

\begin{tabular}{lllllll}
\hline Parameters & \multicolumn{3}{l}{ 1 Month } & & \multicolumn{3}{l}{3 Months } & $P$ \\
\cline { 2 - 3 } & Mean & SD & & Mean & SD & \\
\hline MTF $_{\text {cutoff }}$ (cpd) & 38.49 & 10.35 & & 37.64 & 9.74 & 0.475 \\
Strehl2D Ratio & 0.22 & 0.07 & 0.21 & 0.06 & 0.587 \\
OV100\% & 1.28 & 0.35 & 1.25 & 0.32 & 0.475 \\
OV20\% & 1.30 & 0.43 & 1.25 & 0.39 & 0.408 \\
OV9\% & 1.30 & 0.47 & & 1.24 & 0.44 & 0.401 \\
OSI & 1.00 & 0.83 & & 0.98 & 0.71 & 0.954 \\
\hline
\end{tabular}

MTF cutoff modulation transfer function cutoff frequency, cpd cycles per degree, Streh/2D Ratio Strehl in two dimensions ratio, OV optical quality analysis system value, OSI objective scatter index, SD standard deviation

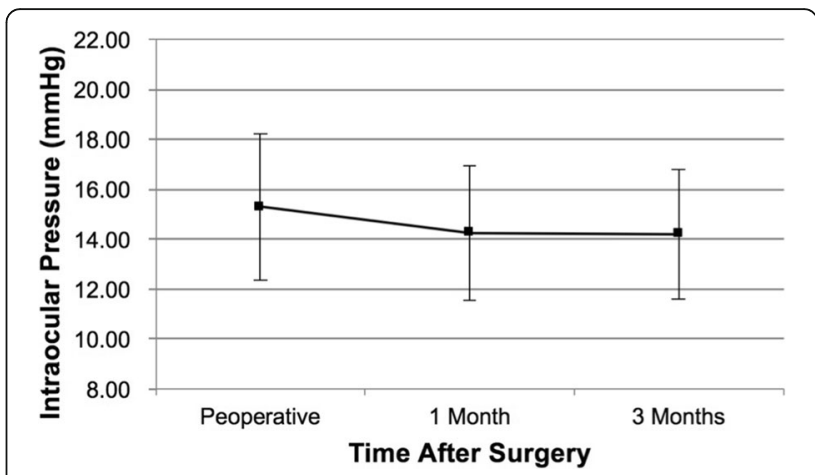

Fig. 2 Intraocular pressure after ICL V4C implantation

visual quality $[16,19,24,26-28]$. Tian et al. found that the two kinds of ICL had similar efficacy of visual quality for high myopia, and also similar results in low-order aberrations, while high-order aberrations and spherical aberrations were higher after ICL V4c implantation [28]. In Kamiya's study, the two ICLs produced similar optical quality and intraocular scattering results [24]. Kamiya et al. found that the mean OSI was 0.90 in 201 myopic adults with SE values ranging from -1.25 to $-8.25 \mathrm{D}$, and that contrast sensitivity increased when OSI decreased [29]. Our previous study found that the mean $\mathrm{MTF}_{\text {cutoff }}$ was $35.15 \mathrm{cpd}$ and the mean OSI was 0.74 in 274 myopic adults with SE values ranging from -0.63 to $-14.25 \mathrm{D}$ [11]. In the current study, the mean $\mathrm{MTF}_{\text {cutoff }}$ was $38.20 \mathrm{cpd}$ and the mean OSI was 1.00 at 3 months, indicating high optical quality after ICL V4c implantation. Kamiya et al. and Huseynova et al. investigated optical quality at 3 months after ICL V4c implantation. The mean postoperative $\mathrm{MTF}_{\text {cutoff, }}$ Strehl2D ratio, and OSI were $26.21 \mathrm{cpd}$, 0.16 , and 1.16, respectively, in Kamiya et al.'s study [24], and the postoperative mean OSI was 1.08 in Huseynova et al.'s study [19]. When compared with the above two studies, retinal image quality and intraocular scattering were approximately higher and lower, respectively, in our study. This difference might be due to regional and ethnic differences, however, the reason needs to be explored further.

The identical optical quality results at 1 and 3 months suggested that the optical system could achieve stability shortly after ICL V4c implantation. In contrast, optical quality sometimes decreases after PRK and LASIK surgeries [30]. In our previous studies on optical quality after small incision lenticule extraction (SMILE) to correct moderate to high myopia correction, a temporary increase in intraocular scattering in the early stage ( 3 months postoperatively) was observed before the optical quality stabilized $[10,12]$. The pIOL implantation procedures do not interfere with the central cornea and maintain the prolate shape of the cornea; thus, it should induce less high order aberrations than the corneal refractive surgeries and avoid the corneal wound healing process of the central optical 
zone in myopia correction [31-36]. These factors could contribute to the high stability and level of optical quality after ICL V4c implantation.

The stable IOP indicates that the central hole in ICL V4c could help maintain the IOP without peripheral iridotomy, which further certified the safety of ICL V4c implantation. As of yet, no study has reported secondary cataract after ICL V4c implantation [3, 4, 16-20]. ICL V4c improves the aqueous humor circulation to the anterior surface of the crystalline lens, thus reducing the risk of cataract formation [7-9]. Alfonso et al. reported that, for conventional ICLs, the mean time between phakic intraocular lens implantation and cataract surgery was $4.2 \pm 1.8$ years [5]. Therefore, longer-term observations of the above-mentioned parameters are needed for a more comprehensive understanding of ICL V4c implantation. In the present study, ocular scattering was measured with a double-pass system, which was suggested to be a useful tool in the preoperative evaluation of patients with early cataract [37]. Thus, OSI might be used as an objective parameter to monitor early cataract formation after ICL V4c implantation in further studies.

\section{Conclusions}

In this 3-month prospective study, ICL V4c implantation was shown to be a safe, effective and stable way to correct high myopia. ICL V4c implantation has little influence on IOP. Patients could achieve high, stable postoperative optical quality shortly after the surgery.

\begin{abstract}
Abbreviations
CDVA: Corrected distance visual acuity; cpd: Cycles per degree; D: Diopters; ICL V4c: Posterior chamber phakic implantable collamer lens with a central hole; IOP: Intraocular pressure; logMAR: Logarithm of the minimal angle of resolution; MTF cutoff: Modulation transfer function cutoff frequency; N: Number of eyes; NCT: Non-contact tonometer; OQAS II: Double-pass optical quality analysis system; OSI: Objective scatter index; OV: Optical quality analysis system value; SD: Standard deviation; SE: Spherical equivalent; SPSS: Statistical package for the social sciences; Strehl2D Ratio: Strehl in 2 dimensions ratio; UDVA: Uncorrected distance visual acuity
\end{abstract}

\section{Acknowledgments}

The authors thank Lin Wang and Mingzi Piao of the Eye Department of EENT Hospital for their helpful advice. We would also like to thank all the patients participating in the study. This research was supported by the Natural Science Foundation of China for Young Scholars (Grant No. 81700872), the National Natural Science Foundation of China (Grant No. 81570879, No. 81770955), the Outstanding Academic Leaders Program of Shanghai Health System (Grant No. XBR2013098), the Shanghai Shenkang Hospital Development Center (Grant No. SHDC12016207), and the Health and Family Planning Committee of Pudong New District of Shanghai, China (Grant No. PW2014D-1)

\section{Availability of data and materials}

Data and materials are available upon request from the corresponding author at doctzhouxingtao@163.com and doctxiaoyingwang@163.com.

\section{Authors' contributions}

Literature screening and selection was performed by HM and XC. XW and XZ participated in the design of the study. HM and XC drafted the manuscript. HM and XC carried out the statistical analysis. MT and YC prepare and review of the manuscript. XW and XZ has given final approval of the version to be published. All authors read and approved the final manuscript.

\section{Ethics approval and consent to participate}

This study was conducted in accordance with the principles of the Declaration of Helsinki and was approved by the Ethics Committee of the Eye and ENT Hospital Review Board of Fudan University. Written informed consent was obtained from all patients after the nature and possible consequences of the study were explained.

\section{Competing interests}

The authors declare that they have no competing interests.

\section{Publisher's Note}

Springer Nature remains neutral with regard to jurisdictional claims in published maps and institutional affiliations.

Received: 18 November 2017 Accepted: 30 May 2018

Published online: 14 June 2018

\section{References}

1. Brenner LF, Alio JL, Vega-Estrada A, Baviera J, Beltran J, et al. Clinical grading of post-LASIK ectasia related to visual limitation and predictive factors for vision loss. J Cataract Refract Surg. 2012;38:1817-26.

2. Kamiya K, Shimizu K, Kobashi H, Igarashi A, Komatsu M, et al. Three-year follow-up of posterior chamber toric phakic intraocular lens implantation for the correction of high myopic astigmatism in eyes with keratoconus. $\mathrm{Br} \mathrm{J}$ Ophthalmol. 2015;99:177-83.

3. Gonzalez-Lopez F, Bilbao-Calabuig R, Mompean B, de Rojas V, Luezas J, et al. Intraocular pressure during the early postoperative period after 100 consecutive implantations of posterior chamber phakic intraocular lenses with a central hole. J Cataract Refract Surg. 2013;39:1859-63.

4. Higueras-Esteban A, Ortiz-Gomariz A, Gutierrez-Ortega R, Villa-Collar C, Abad-Montes JP, et al. Intraocular pressure after implantation of the visian implantable collamer lens with CentraFLOW without iridotomy. Am J Ophthalmol. 2013;156:800-5.

5. Alfonso JF, Lisa C, Fernandez-Vega L, Almanzar D, Perez-Vives C, et al. Prevalence of cataract after collagen copolymer phakic intraocular lens implantation for myopia, hyperopia, and astigmatism. J Cataract Refract Surg. 2015;41:800-5.

6. Sanders DR. Anterior subcapsular opacities and cataracts 5 years after surgery in the visian implantable collamer lens FDA trial. J Refract Surg. 2008;24:566-70

7. Kawamorita T, Uozato H, Shimizu K. Fluid dynamics simulation of aqueous humour in a posterior-chamber phakic intraocular lens with a central perforation. Graefes Arch Clin Exp Ophthalmol. 2012;250:935-9.

8. Shiratani T, Shimizu K, Fujisawa K, Uga S, Nagano K, et al. Crystalline lens changes in porcine eyes with implanted phakic IOL (ICL) with a central hole. Graefes Arch Clin Exp Ophthalmol. 2008;246:719-28.

9. Fujisawa K, Shimizu K, Uga S, Suzuki M, Nagano K, et al. Changes in the crystalline lens resulting from insertion of a phakic $\mathrm{IOL}(\mathrm{ICL})$ into the porcine eye. Graefes Arch Clin Exp Ophthalmol. 2007;245:114-22.

10. Miao H, He L, Shen Y, Li M, Yu Y, et al. Optical quality and intraocular scattering after femtosecond laser small incision lenticule extraction. $J$ Refract Surg. 2014;30:296-302.

11. Miao H, Tian M, He L, Zhao J, Mo X, et al. Objective optical quality and intraocular scattering in myopic adults. Invest Ophthalmol Vis Sci. 2014; 55:5582-7.

12. Miao H, Tian M, Xu Y, Chen Y, Zhou X. Visual outcomes and optical quality after femtosecond laser small incision Lenticule extraction: an 18-month prospective study. J Refract Surg. 2015;31:726-31.

13. Martinez-Roda JA, Vilaseca M, Ondategui JC, Giner A, Burgos FJ, et al. Optical quality and intraocular scattering in a healthy young population. Clin Exp Optom. 2011;94:223-9.

14. Kamiya K, Umeda K, Kobashi H, Shimizu K, Kawamorita T, et al. Effect of aging on optical quality and intraocular scattering using the double-pass instrument. Curr Eye Res. 2012;37:884-8.

15. Artal P, Benito A, Perez GM, Alcon E, De Casas A, et al. An objective scatter index based on double-pass retinal images of a point source to classify cataracts. PLoS One. 2011;6:e16823.

16. Shimizu K, Kamiya K, Igarashi A, Shiratani T. Intraindividual comparison of visual performance after posterior chamber phakic intraocular lens with and without a central hole implantation for moderate to high myopia. Am J Ophthalmol. 2012;154:486-94. 
17. Shimizu K, Kamiya K, Igarashi A, Shiratani T. Early clinical outcomes of implantation of posterior chamber phakic intraocular lens with a central hole (hole ICL) for moderate to high myopia. Br J Ophthalmol. 2012;96:409-12.

18. Lisa C, Naveiras M, Alfonso-Bartolozzi B, Belda-Salmeron L, Montes-Mico R, et al. Posterior chamber collagen copolymer phakic intraocular lens with a central hole to correct myopia: one-year follow-up. J Cataract Refract Surg. 2015:41:1153-9.

19. Huseynova T, Ozaki S, Ishizuka T, Mita M, Tomita M. Comparative study of 2 types of implantable collamer lenses, 1 with and 1 without a central artificial hole. Am J Ophthalmol. 2014;157:1136-43.

20. Alfonso JF, Lisa C, Fernandez-Vega CL, Belda-Salmeron L, Madrid-Costa D, et al. Clinical outcomes after implantation of a posterior chamber collagen copolymer phakic intraocular lens with a central hole for myopic correction. J Cataract Refract Surg. 2013;39:915-21.

21. Uozato H, Shimizu K, Kawamorita T, Ohmoto F. Modulation transfer function of intraocular collamer lens with a central artificial hole. Graefes Arch Clin Exp Ophthalmol. 2011;249:1081-5.

22. Perez-Vives C, Ferrer-Blasco T, Madrid-Costa D, Garcia-Lazaro S, Montes-Mico R. Optical quality comparison of conventional and hole-visian implantable collamer lens at different degrees of decentering. Am J Ophthalmol. 2013; 156:69-76.

23. Perez-Vives C, Ferrer-Blasco T, Madrid-Costa D, Garcia-Lazaro S, Montes-Mico R. Visual quality comparison of conventional and hole-visian implantable collamer lens at different degrees of decentering. Br J Ophthalmol. 2014;98:59-64.

24. Kamiya K, Shimizu K, Saito A, Igarashi A, Kobashi H. Comparison of optical quality and intraocular scattering after posterior chamber phakic intraocular lens with and without a central hole (hole ICL and conventional ICL) implantation using the double-pass instrument. PLoS One. 2013;8:e66846.

25. Xun C, H Miao, Naidu RK, Xiaoying W, et al. Comparison of early changes in and factors affecting vault following posterior chamber phakic implantable collamer lens implantation without and with a central hole (ICL V4 and ICL V4C). BMC Ophthalmol. 2016;16:161

26. Hyun J, Lim DH, Eo DR, Hwang S, Chung ES, Chung TY. A comparison of visual outcome and rotational stability of two types of toric implantable collamer lenses (TICL) : V4 versus V4C. PLoS One. 2017;12:e0183335.

27. Shimizu K, Kamiya K, Igarashi A, Kobashi H. Long-term comparison of posterior chamber phakic intraocular lens with and without a central hole (hole ICL and conventional ICL) implantation for moderate to high myopia and myopic astigmatism: consort-compliant article. Medicine (Baltimore). 2016;95:e3270.

28. Tian Y, Jiang HB, Jiang J, Wen D, Xia XB, Song WT. Comparison of implantable Collamer Lens Visian ICL V4 and ICL V4C for high myopia: a cohort study. Medicine (Baltimore). 2017;96:e7294.

29. Kamiya K, Shimizu K, lijima A, Kobashi H. Factors influencing contrast sensitivity function in myopic eyes. PLoS One. 2014;9:e113562.

30. Ondategui JC, Vilaseca M, Arjona M, Montasell A, Cardona G, et al. Optical quality after myopic photorefractive keratectomy and laser in situ keratomileusis: comparison using a double-pass system. J Cataract Refract Surg. 2012;38:16-27.

31. Sanders $D$, Vukich JA. Comparison of implantable collamer lens $(\mathrm{ICL})$ and laser-assisted in situ keratomileusis (LASIK) for low myopia. Cornea. 2006;25: 1139-46.

32. Igarashi A, Kamiya K, Shimizu K, Komatsu M. Visual performance after implantable collamer lens implantation and wavefront-guided laser in situ keratomileusis for high myopia. Am J Ophthalmol. 2009:148:164-70.

33. Kamiya K, Igarashi A, Shimizu K, Matsumura K, Komatsu M. Visual performance after posterior chamber phakic intraocular lens implantation and wavefront-guided laser in situ keratomileusis for low to moderate myopia. Am J Ophthalmol. 2012;153:1178-86. el

34. Sanders DR. Matched population comparison of the Visian implantable Collamer Lens and standard LASIK for myopia of -3.00 to -7.88 diopters. J Refract Surg. 2007;23:537-53.

35. Sanders DR, Vukich JA. Comparison of implantable contact lens and laser assisted in situ keratomileusis for moderate to high myopia. Cornea. 2003; 22:324-31.

36. Parkhurst GD. A prospective comparison of phakic collamer lenses and wavefront-optimized laser-assisted in situ keratomileusis for correction of myopia. Clin Ophthalmol. 2016;10:1209-15.

37. Cabot F, Saad A, McAlinden C, Haddad NM, Grise-Dulac A, et al. Objective assessment of crystalline lens opacity level by measuring ocular light scattering with a double-pass system. Am J Ophthalmol. 2013;155:629-35. 631-35

\section{Ready to submit your research? Choose BMC and benefit from:}

- fast, convenient online submission

- thorough peer review by experienced researchers in your field

- rapid publication on acceptance

- support for research data, including large and complex data types

- gold Open Access which fosters wider collaboration and increased citations

- maximum visibility for your research: over $100 \mathrm{M}$ website views per year

At BMC, research is always in progress.

Learn more biomedcentral.com/submissions 